\title{
How accurate are cancer cell lines?
}

\section{Some argue that tumour cells obtained directly from patients are the best way to study cancer genomics.}

For decades, cancer cell cultures grown in Petri dishes have been the foundation of cancer biology and the quest for drug treatments. But now that biologists are exploring cancer genomes, some are asking whether they should pursue a more expensive, less proven strategy that may give a truer picture of key mutations: sequencing cells from tumours plucked directly from patients.

Of the first six cancer genome sequences to be published, three have come from established cell lines and three from primary tumours. Hundreds more sequences are expected soon, both from individual labs and from major efforts such as the Cancer Genome Atlas in Bethesda, Maryland. And although most researchers continue to have a foot in both camps, the atlas project excludes work on cell lines.

The major criticism of cell lines is that not all cancer types can be grown indefinitely in the laboratory. Those that do grow differ genetically from primary tissue, accumulating new mutations as they adapt to their artificial environment. When implanted in rodents, brain-cancer cell lines tend to form a 'bowling ball' mass of cells rather than infiltrating the brain like a spider web, as they do in humans, says Howard Fine, head of neuro-oncology at the National Institutes of Health in Bethesda. "The glioma [brain cancer] cell lines we've been using bear so little resemblance to what happens in people, it's no wonder that when we screen therapeutics on animals and bring them to the clinic they come back negative," he says.

Cell lines for other cancer types may resemble primary tumours more closely, but some teams still aren't interested. "To be honest, I'm not sure why you would ever want to sequence cell lines," says Timothy Graubert at Washington University in St Louis, Missouri. Graubert was an author on the first cancer genome to be published $^{1}$, which was based on samples from a patient with acute myeloid leukaemia. "We feel the arguments in favour of using primary cells are compelling," he says.

But primary cells are more expensive to sequence because, unlike cell lines, they contain a mixture of genetically distinct cells, so researchers need to use costly techniques to pinpoint rare mutations that are not spread uniformly throughout the tumour. More crucially, says Stan Nelson, an oncologist at

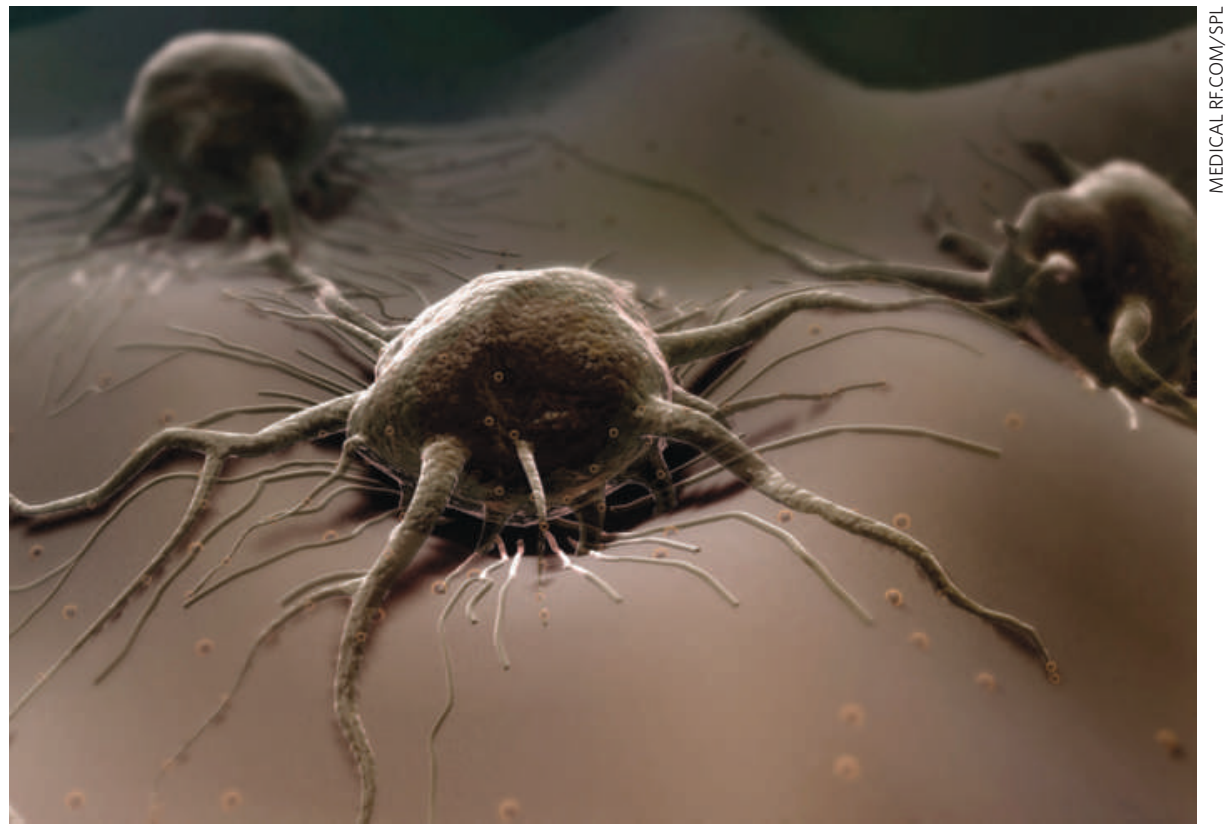

Cultured cancer cells might have different genetic characteristics from in situ tumours.

the University of California, Los Angeles, it will take thousands of comparisons between tumour sequences and normal DNA to equal the benefit of sequencing the top 100 cell lines, which are backed by a wealth of literature on their development and biology. And, so far, the results from genomic studies of primary tumours haven't impressed some cancer biologists. For example, most of the mutations that Graubert and his colleagues identified by comparing sequences from the tumour cells with normal tissue from the same individual turned out not to be significant, says Nelson. His group has sequenced the widely used glioma cell line U87MG (ref. 2), but he says there's value in both approaches.

\section{Down but not out}

This week, two studies in Nature ${ }^{3,4}$ highlight the continued relevance of cell lines. Researchers led by pathologist Michael Stratton of the Wellcome Trust Sanger Institute in Cambridge, $\mathrm{UK}$, analysed deletion mutations in 746 cell lines, including the 59 'NCI-60' lines that have been used in developing a wide range of cancer drugs $^{3}$. Cell lines don't contain the non-cancerous cells found in primary tumours, making the cultivated lines ideal for finding deletions in the cancer genome, says Stratton. His group can then distinguish between segments of the genome that are lost as a result of breakage from those in which a tumour-suppressing gene has lost its functionality.

The second study, led by Matthew Meyerson of the Dana-Farber Cancer Institute in Boston, Massachusetts, searched for deletions and additions of chunks of DNA in 3,131 cancer specimens, including 541 cell lines. He says that the common alterations in cell lines, which include gains and losses of entire arms of chromosomes, are often the same ones found in primary tissue.

That's one reason why Meyerson takes a pragmatic view about the best way to study cancer genomes. Sequencing cancer cell lines makes sense in the short term, he says, but "millions and millions of people are getting cancer, and over time the statistical power of looking at primary tumours is going to be greater". This month, for instance, the Cancer Genome Atlas is shipping 200 colon-cancer samples to sequencing centres, one of 20 tumour types to be sequenced over the next five years.

\section{Brendan Borrell}

1. Ley, T. J. et al. Nature $456,66-72$ (2008).

2. Clark, M. J. et al. PLoS Genet. 6, e1000832 (2010).

3. Bignell, G. R. et al. Nature 463, 893-898 (2010).

4. Beroukhim, R. et al. Nature 463, 899-905 (2010) 